\title{
Autism-associated synaptic vesicle transcripts are differentially expressed in maternal plasma exosomes of physiopathologic pregnancies
}

\author{
Yangwu Fang ${ }^{1,2}$, Chan Wan ${ }^{2}$, Youlu Wen ${ }^{3}$, Ze Wu ${ }^{2}$, Jing Pan ${ }^{2}$, Mei Zhong ${ }^{1 *}$ and Nanbert Zhong ${ }^{2,4^{*}}$
}

\begin{abstract}
Background: During intrauterine development, the formation and function of synaptic vesicles (SVs) are thought to be fundamental conditions essential for normal development of the brain. Lacking advanced technology during the intrauterine period, such as longitudinal real-time monitoring of the SV-associated transcripts (SVATs), which include six pairs of IncRNA-mRNA, has limited acquisition of the dynamic gene expression profile (GEP) of SVATs. We previously reported the differential expression of SVATs in the peripheral blood of autistic children. The current study was designed to determine the dynamic profiles of differentially-expressed SVATs in circulating exosomes (EXs) derived from autistic children and pregnant women at different gestational ages.
\end{abstract}

Methods: Blood samples were collected from autistic children and women with variant physiopathologic pregnancies. EXs were isolated with an ExoQuick Exosome Precipitation Kit and characterized by transmission electron microscopy (TEM), nanoparticle tracking analysis (NTA), and Western blotting. The expression of IncRNAs and IncRNAtargeted mRNAs were quantified using real-time PCR.

Results: SVAT-associated IncRNAs-mRNAs were detected in autistic children and differentially expressed from the first trimester of pregnancy to the term of delivery. Pathologic pregnancies, including spontaneous preterm birth (sPTB), preeclampsia (PE), and gestational diabetes mellitus (GDM), were compared to normal physiologic pregnancies, and shown to exhibit specific correlations between SVAT-IncRNA and SVAT-mRNA of STX8, SLC18A2, and SYP with SPTB; SVAT-IncRNA and SVAT-mRNA of STX8 with PE; and SVAT-IncRNA and SVAT-mRNA of SV2C as well as SVAT-mRNA of SYP with GDM.

Conclusion: Variant complications in pathologic pregnancies may alter the GEP of SVATs, which is likely to affect the intrauterine development of neural circuits and consequently influence fetal brain development.

Keywords: Synapse, Spontaneous preterm birth, Preeclampsia, Gestational diabetes mellitus, Exosomes

\footnotetext{
*Correspondence: zhongmei@smu.edu.cn; nambert.zhong@opwdd.ny.gov ${ }^{1}$ Department of Obstetrics and Gynecology, Nanfang Hospital, Southern Medical University, 1838 North Guangzhou Avenue, Guangzhou 510515, China

${ }^{4}$ Department of Human Genetics, New York State Institute for Basic Research in Developmental Disabilities, 1050 Forest Hill Rd, Staten Island, NY 10314, USA

Full list of author information is available at the end of the article
}

\section{Background}

Synapses, which are composed of presynaptic and posterior synapses, are specialized junctions between neurons in the brain that relay and process information. Synapses connect neurons into the intricate neural network of neural circuits, which are the fundamental elements that induce brain function [1]. The concept of neural circuits

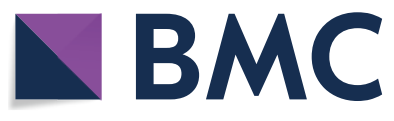

(c) The Author(s) 2021. This article is licensed under a Creative Commons Attribution 4.0 International License, which permits use, sharing, adaptation, distribution and reproduction in any medium or format, as long as you give appropriate credit to the original author(s) and the source, provide a link to the Creative Commons licence, and indicate if changes were made. The images or other third party material in this article are included in the article's Creative Commons licence, unless indicated otherwise in a credit line to the material. If material is not included in the article's Creative Commons licence and your intended use is not permitted by statutory regulation or exceeds the permitted use, you will need to obtain permission directly from the copyright holder. To view a copy of this licence, visit http://creativeco mmons.org/licenses/by/4.0/. The Creative Commons Public Domain Dedication waiver (http://creativecommons.org/publicdomain/ zero/1.0/) applies to the data made available in this article, unless otherwise stated in a credit line to the data. 
serves as a helpful tool to probe into the mechanism underlying brain function; however, it is crucial to take into consideration the limitations of the concept when assessing the function of synapses in neurologic processing. The coexistence of electrical and chemical synapses in the brain structures of most animals has been longestablished, but investigation into the details of these two transmission modalities and the distribution are ongoing [2]. Chemical transmission involves complex presynaptic molecular machinery that controls the release of neurotransmitters in an orderly manner when an action potential is fired at the synaptic terminal. Thus far, exploration of the mechanisms underlying chemical transmission forms the central focus of most research [3]. The process of synaptic pruning is at the heart of our understanding of circuit constructions. Synaptic vesicles (SVs) are small carriers that are electron-lucent at presynaptic terminals. Neurotransmitters accumulate in SVs, awaiting release through $\mathrm{Ca}^{2+}$-triggered exocytosis [4]. SVs contain synaptic vesicle-associated transcripts (SVATs) and synaptic vesicle-associated proteins (SVAPs), which have a variety of functions, including the following functions: ion channels; scaffolding proteins; neurotransmitter receptors; and facilitating synaptic cell adhesion. SVs are produced locally at synaptic terminals and are regenerated through SV cycling (SVC) that contains proteins with two functions (the uptake of neurotransmitters and membrane trafficking [5]), thus supporting both synaptic functions and structural integrity. Few studies, however, have investigated the mechanisms that induce synaptic neurotransmission and the relationship with many neurologic diseases [6].

Extracellular vesicles (EVs) are nano particles that consist of a lipid bilayer membrane and RNA, DNA, and proteins. EVs can be separated from any biological fluid and can be used to reflect the balance between uptake and secretion by different types of local cells. EVs are comprised of a variety of biomolecules, many of which are potential biomarkers for disease [7]. Exosomes (EXs), defined as small spherical EVs that are $30-150 \mathrm{~nm}$ in size, are a class of EVs derived from the endosome/multivesicular body system. EXs are responsible for selectively transferring biologically-active materials between cells, i.e., EXs are involved in intercellular communications. Recent findings have demonstrated the transport and transfer of proteins and miRNA via EXs, indicating that EXs are potential effectors in diseases and innovative biomarkers for diagnosing, predicting prognoses, and treating diseases [8].

RNA participates in numerous biological activities. Many types of RNA exist within the human body, among which messenger RNA (mRNA, with an average length of 1000-1500 nucleotides), microRNA (miRNA, with an average length of $\sim 20$ nucleotides), and long noncoding RNA (lncRNA, with an average length $\geq 200$ nucleotides) are the most widely investigated as a result of the wide distribution [9]. Recently, lncRNAs have been shown to be present in genomes as intronic, antisense, or large intergenic non-coding RNAs (ncRNAs). In addition, lncRNAs are located at regions associated with promoters or untranslated, functioning as cis- or transregulators. LncRNAs may serve as a transcriptional and post-transcriptional regulators of neural development and cell differentiation, hence lncRNAs are related to many human neural disorders [10], including psychiatric and neurodegenerative disorders, immune dysfunction, autoimmune diseases, cardiovascular disease, cancer, and reproductive disorders [11]. LncRNA deregulation is gaining recognition because many pathologic processes bear this characteristic. Moreover, lncRNAs associated with malignant tumors may be utilized as diagnostic or prognostic biomarkers, and potentially as targets of selective silencing of innovative therapeutic agents [12].

EXs are produced by every cell type within the central nervous system, these including basic neurons, oligodendrocytes, microglia, astrocytes [13], and neural stem cells. Neuron-derived EXs can be detected in peripheral blood plasma [14]. Fetal EXs can also enter the maternal circulatory system via the placenta [15]. Therefore, detecting different components of fetal EXs in the maternal blood circulation can provide new indicators of prenatal fetal development, including fetal brain development. In our previous sequencing studies on autism in children, altered expression of lncRNAs and mRNAs was documented to be associated with SV trafficking [16]. The present study aimed to investigate the differentiallyexpressed patterns of lncRNA-mRNA SVATs in plasma EXs from children and maternal peripheral blood at different gestational ages. The differences in expression of these molecules between EXs of healthy pregnant women and women with pathologic pregnancies, including spontaneous preterm birth (sPTB) resulting from spontaneous preterm labor (sPTL) or preterm premature rupture of membrane (pPROM), preeclampsia (PE), or gestational diabetes mellitus (GDM), were assessed to identify molecular candidates as early predictive biomarkers.

\section{Methods \\ Sample collection}

This study was approved by the Medical Ethics Committee of Guangdong Triple-Nine Brain Hospital (recruitment of 14 children with autism spectrum disorder [ASD] and 14 healthy children, 2-10 years of age) and Nanfang Hospital of Southern Medical University (recruitment of pregnant women). Informed consent was obtained from the pregnant women and the guardians of 
the children. The peripheral blood of pregnant women was collected, as follows: $24^{+0}-32^{+6}$ gestational weeks (GWs $[n=20])$; and $33^{+0}-39^{+6}$ GWs $(n=20)$. EXs were isolated from plasma samples and subjected to RNAseq. A minimum of 30 healthy pregnancies collected on a weekly basis at each GW from the $11^{\text {th }}-40^{\text {th }} \mathrm{GW}$ were subjected to SVATs assessment. Plasma from 38 sPTBs, 34 women with GDM, and 19 women with PE in the third trimester (34 GW for sPTB and 34-35 GW for PE and GDM) were retrospectively used as case-controls.

\section{EX isolation}

Peripheral blood was collected into EDTA tubes (BD, New York City, NY, USA). After the blood was collected, the tubes were inverted five times, stored on ice, and processed within $30 \mathrm{~min}$. Each specimen was centrifuged at $1500 \mathrm{~g}$ for $15 \mathrm{~min}$ at $4{ }^{\circ} \mathrm{C}$ to separate plasma from cells. The plasma was stored at $-80{ }^{\circ} \mathrm{C}$ until processing of EX isolation, for which $550 \mu \mathrm{L}$ of plasma was incubated with $5.5 \mu \mathrm{L}$ of thrombin (TMEXO-1; System Biosciences, Mountain View, CA, USA) at room temperature for $5 \mathrm{~min}$, then centrifuged in a microcentrifuge at $10,000 \mathrm{~g}$ for $5 \mathrm{~min}$. Approximately $500 \mu \mathrm{L}$ of supernatant was transferred into a sterile vessel and incubated with $120 \mu \mathrm{L}$ of ExoQuick Exosome Precipitation Solution (EXOQ5TM; System Biosciences) and $5.5 \mu \mathrm{L}$-cocktails of protease and phosphatase inhibitors (PM6020; Huabodeyi, Beijing, China) at $4{ }^{\circ} \mathrm{C}$ for $30 \mathrm{~min}$, followed by centrifugation at $300 \mathrm{~g}$ for $30 \mathrm{~min}$ at $4{ }^{\circ} \mathrm{C}$. EX pellets were resuspended in $100 \mu \mathrm{L}$ of PBS (Gibco, Grand Island, NY, USA) and stored at $-80^{\circ} \mathrm{C}$ until RNA was isolated. The concentration of total EXs was determined using a BCA Protein Assay (Thermo Fisher, Waltham, MA, USA).

\section{Transmission electron microscopy}

Pellets of freshly-isolated EXs suspended in PBS buffer were dropped onto a carbon-coated copper grid, dried, then stained with $1 \%$ uranyl acetate (Sigma-Aldrich, St. Louis, MO, USA) for $5 \mathrm{~min}$. The grids were imaged under a Hitachi H-7650 transmission electron microscope (Hitachi, Tokyo, Japan).

\section{Nanoparticle tracking analysis (NTA)}

An aliquot of the EX suspension $(10 \mu \mathrm{L}$ of vesicles in 90 $\mu \mathrm{L}$ of buffer) was collected after adding $10 \mu \mathrm{L}$ of $10 \%$ glycine, which was then diluted with PBS to a final ratio of 1:200 before lysis with Mammalian Protein Extraction Reagent (Millipore, Billerica, MA, USA). The mean concentration (particles/mL) and diameter $(\mathrm{nm})$ of EXs were calculated using NTA 3.1 nanoparticle tracking software and the Nanosight NS300 system with a G532-nm laser module (Malvern Instruments, Malvern, UK).

\section{Western blotting}

To confirm the success of EX isolation, 30-40 $\mu \mathrm{g}$ of exosomal protein was loaded onto a polyacrylamide gel (5\% stacking and $10 \%$ resolving gel). After running the gel at $90 \mathrm{~V}$ for $20 \mathrm{~min}$, followed by $120 \mathrm{~V}$ for $60 \mathrm{~min}$ in running buffer, the proteins were transferred to a polyvinyl fluoride membrane $(0.45 \mu \mathrm{m}$; Millipore $)$ and blocked with $5 \%$ skim milk (BD, Lincoln, NE, USA) for $2 \mathrm{~h}$. The membranes were incubated overnight with primary antibodies (Abs) against CD63, CD9, C81 (rabbit pAb; SBI, Palo Alto, CA, USA), pregnancy-specific globin (PSG1), nestin (NES), progesterone receptor [PGR] (rabbit pAb; Absin, Shanghai, China), L1 cell adhesion molecule [L1CAM] (rabbit pAb; Abcam, Cambridge, UK), and placental alkaline phosphatase [PLAP] (mouse mAb; Santa Cruz Biotechnology, Inc., Santa Cruz, $\mathrm{CA}$, USA) at $4{ }^{\circ} \mathrm{C}$. The $\mathrm{Ab}$ dilution ranged from $500-$ to 1000 -fold times, as appropriate. The next day, the membranes were washed and incubated with secondary antibodies (goat anti-rabbit-HRP; Beyotime, Shanghai, China). The blotted proteins were visualized using an enhanced chemiluminescence kit (Pierce Biotechnology, Rockford, IL, USA), and the signal was detected using Saga (Sage Sciences, Lincoln, NE, USA).

\section{RNA extraction and quantitative real-time PCR}

Total RNA extraction from EXs was achieved using TRIzol (Invitrogen, Carlsbad, CA, USA). The HiScript Reverse Transcription Kit and ChamQ SYBR Green qPCR Kit (Vazyme, Nanjing, China) were used to perform reverse transcription and quantify RNAs in accordance with the manufacturer's instructions. The levels of lncRNA-targeted mRNA and lncRNA expression were established using quantitative real-time PCR (the primer sequences used in quantitative PCR are listed in Additional file 1: Tables S1) using a LightCycle 96 system (Roche, Grand Island, NY, USA). U6 RNA was utilized as a control for the numerical analysis. The quantity of mRNA and lncRNA was normalized to the U6 level. Triplet quantitative measurement for each lncRNA or mRNA was performed. A relative normalized expression of each sample was determined based on the level of sample expression at $11 \mathrm{GW}$.

\section{Data analysis}

All triplet analysis data are presented as the mean $\pm \mathrm{SE}$ For the relative IncRNA and mRNA quantification, the means of the control groups were set to 1 , which was not displayed in the drawing. The data were subjected to one-way analysis of variance, followed by an unpaired, two-tailed t-test. Differences were considered 
significant at a $P<0.05$. The figures were drawn with GraphPad Prism (version 8.0).

\section{Results}

Expression of exosomal SVATs in ASD

Previously, we determined the differential expression of SVATs in peripheral blood lymphocytes of children with autism [16]. In the current study, we have successfully replicated the differential expression of six SVAT pairs in peripheral lymphocytes and in circulating EXs isolated from peripheral plasma. As shown in Table 1, SVAT-lncRNAs of SLC18A2, SYT9, STX8, and $S Y T 15$ were measured to be up-regulated, while SVATlncRNAs of $S V 2 C$ and $S Y P$ were minimally expressed compared to healthy children. Differential expression profiles (DEPs) were documented in 6 SVAT-IncRNA

Table 1 GEP of SVAT-IncRNAs and SVAT-mRNAs in autism exosomes

\begin{tabular}{lllllllll}
\hline LncRNA & & & & mRNA & & & \\
Symbol & Blood & DEP & Exosomes & DEP & Symbol & Blood & DEP & Exosomes \\
\hline RP11-501J20.5 & Yes & Up & Yes & Up & SLC18A2 & ND & NA & Yes \\
RP11-38L15.3 & Yes & Up & Yes & Up & SYT15 & Yes & Up & Yes \\
STX8 & Yes & Up & Yes & Up & STX8 & ND & NA & Yes \\
CTD-2516F10.2 & Yes & Up & Yes & Up & SYT9 & ND & NA & Yes \\
RP11-466P24.2 & Yes & Down & Yes & Up & SV2C & ND & NA & Yes \\
SYP-AS1 & Yes & Down & Yes & Down & SYP & ND & NA & Yes \\
STX16-NPEPL1 & Yes & Up & ND & NA & STX16 & ND & NA & ND \\
AC005606.14 & Yes & Up & ND & NA & SYNGR3 & ND & NA & ND \\
RP1-78014.1 & Yes & Up & ND & NA & SYT1 & ND & NA & ND \\
AK128569 & Yes & Up & ND & NA & SYT9 & ND & NA & ND \\
STXBP5-AS1 & Yes & Down & ND & NA & STXBP5 & ND & NA & ND \\
RP5-839b4.7 & Yes & Up & ND & NA & SNAP25 & Yes & Up & ND
\end{tabular}

$D E P$ differential expression profile, Up up-regulated, Down down-regulated, ND not detectable, NA not available, NS not significant
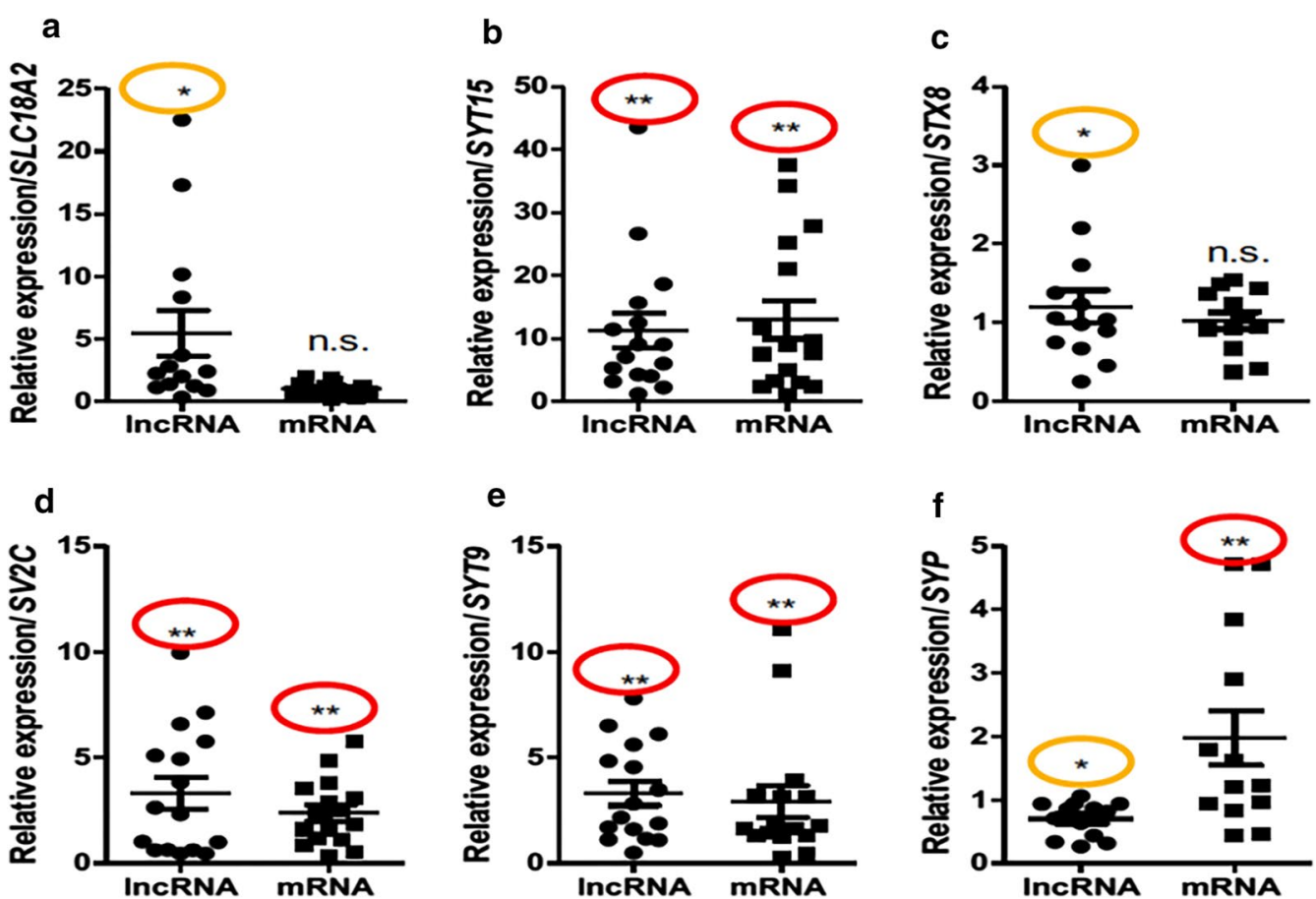

Fig. 1 GEP of exosomal SVATs. Expression of SVATs in circulated exosomes derived from ASD children; compared to healthy children. ${ }^{*} P<0.05$ in orange oval, ${ }^{* *} P<0.01$ in red oval 
loci and SLC18A2, SYT9, STX8, SV2C, SYP, and SYT15 SVAT-mRNA (Fig. 1) when exosomal RNAs of 14 children with ASD were examined. As presented in Fig. 1, three up-regulated SVAT-mRNAs at the SYT15 (Fig. 1b), SYT9 (1E), and SYP loci (1F) and one downregulated SVAT-mRNA at the $S V 2 C$ (1D) locus were identified. We also noticed that 10 of 12 SVAT-associated mRNAs could not be detected from the autistic blood specimens. This finding provided evidence that RNA is very stable in EXs application which may give a better approach for testing biomarkers.

\section{Identification and characterization of EXs isolated from maternal plasma}

EXs were isolated from plasma derived from pregnancies using a commercial kit and observed via transmission electron microscopy. The data showed that the EXs were spherical and wrapped by a double-membrane structure (Fig. 2a). The actual size of the EXs in the NTA particle size analysis system ranged from 50 to $150 \mathrm{~nm}$, with most EXs observed to be $<100 \mathrm{~nm}$ in size (Fig. 2b). This finding was confirmed by antibodies against $\mathrm{CD} 63$, CD9, and CD81, which are considered to be exosomal markers (Fig. 2d, e). Antibody against PSG1 showed that EXs isolated from a pregnant woman (P) were not present in a non-pregnant female (F) and male (M), indicating that the EXs in pregnancy may be derived from pregnancy-specific tissue or cells (Fig. 2d). Indeed, PLAP, a placenta-specific marker, was shown in four EX pregnancy samples (Fig. 2e, [P1-4]). Similarly, the neuron-specific biomarkers, NES (Fig. 2c, e) and L1CAM (Fig. 2c), were also present in EXs. These results demonstrated that EXs isolated from the maternal circulation were heterogeneous and derived from placental trophoblasts as well as neurons.

\section{Discovery study of gene expression profiles (GEPs)} of SVAT-IncRNAs and SVAT-mRNAs in pregnancies

To investigate GEPs of SVATs in physiopathologic pregnancies, a discovery study of SVATs, SVAT-lncRNAs and SVAT-mRNAs, was conducted by RNA-seq at two timepoints of gestational ages, as presented in a heatmap (Fig. 3). Lanes 1 and 2, representing the sPTB group, have significantly different profiles compared with the other groups. The GEP at each lane represents a pool of gene expression from a mixed subset of exosomal RNAs.

\section{GEPs of SVATs during between the 11th and 40th GWs}

After demonstrating that SVATs are expressed in the 3rd trimester of physiopathologic pregnancies, we explored the GEPs of SVATs in earlier stages of pregnancy to determine the longitudinal expression from the $1^{\text {st }}-3^{\text {rd }}$ trimesters of pregnancy. Through continuous analysis of pregnancies from the $5^{\text {th }}-40^{\text {th }} \mathrm{GW}$, the GEPs of exosomal SVAT transcriptions were determined. Exosomal lncRNAs and mRNAs of all 12 pairs of SVATs were not detectable in the early stage of pregnant plasma specimens from the 5th-10th GW (data not shown). Exosomal lncRNA and mRNA of SLC18A2 were detected from the 18th-26th GW and were highly expressed in the 3rd trimester. At the same time, IncRNA of SLC18A2 had an apparent correlation with mRNA (Fig. 4a), unlike STX8. In addition, the level of STX8 IncRNA expression was stable before the $33^{\text {rd }} \mathrm{GW}$, but the mRNA was highly expressed from the 17th-24th GW (Fig. 4b). LncRNA of $S V 2 C$ was highly expressed in the 18th GW, whereas the mRNA had a high expression plateau from the 18th20th GW; there was no correlation between lncRNA and mRNA of $S V 2 C$ (Fig. 4c). The expression peak of exosomal SYP mRNA was from the 13th-23rd GW, followed by high expression from the $31^{\text {st }} \mathrm{GW}$. LncRNA was highly expressed between the 21st and 30th GW (Fig. 4d). Both

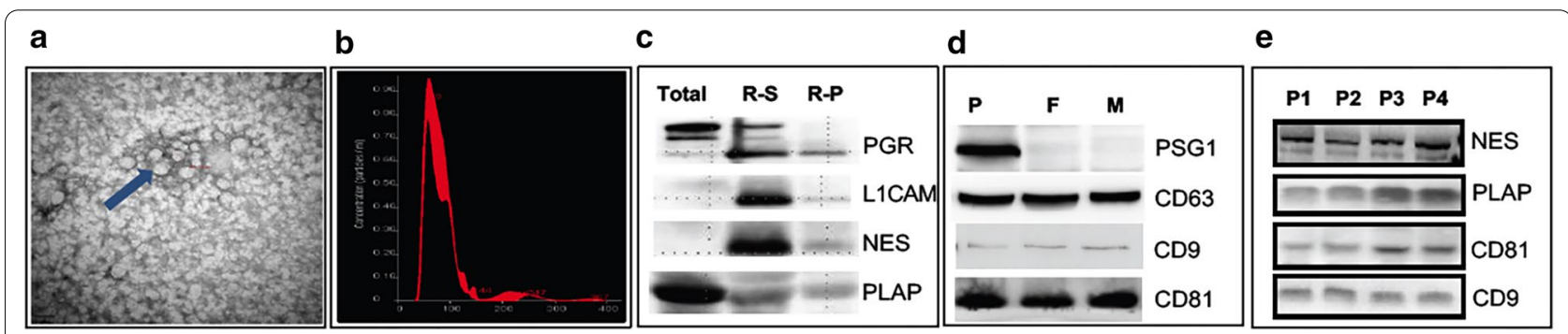

Fig. 2 Isolation of exosomes from peripheral plasmas. Exosomes isolated from maternal plasma were observed under electronic microscopy, as pointed to by an arrow (a) and 50-150 nm in size determined by (b). Proteins loaded from total exosomes (total) and from resuspended supernatant (R-S) or resuspended pellet (R-P) when exosomes treated with lysis buffer during the process of exosomal isolation and probed with antibodies against progesterone receptor (PGR) and placental alkaline phosphatase (PLAP) (c, e). Protein extracted from the exosomes of pregnant women (P), a non-pregnant female (F), and a male (M) were probed with antibodies against pregnant specific globin 1 (PSG1) (d), CD63 (d), and CD9 and CD81 (d, e). The neuro-specific markers, L1CAM (L1 cell adhesion molecule) (c) and nestin (NES), can be detected from plasma exosomes $(\mathbf{c}, \mathbf{e})$ 


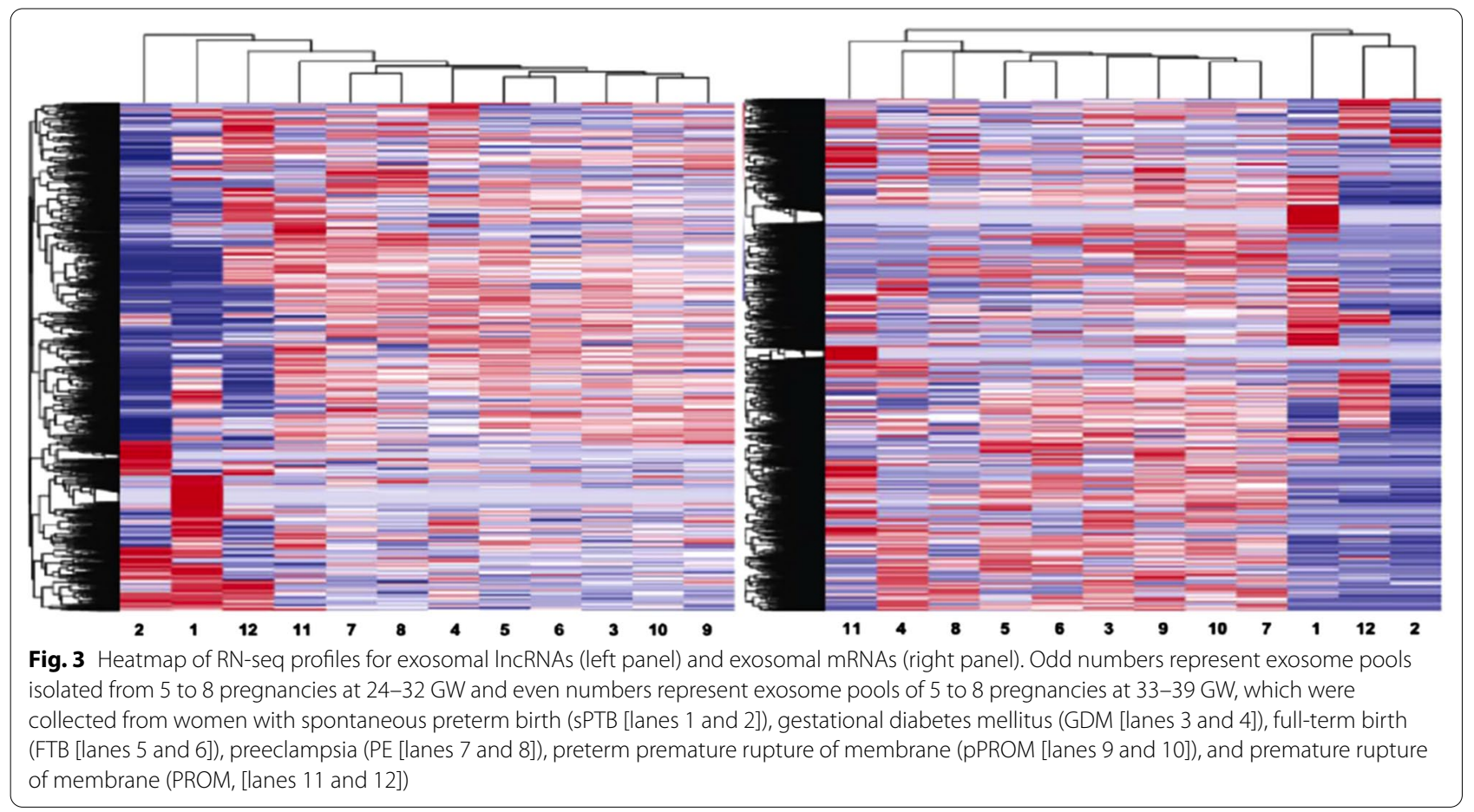

SYT9 IncRNA and mRNA showed an increasing trend at the 18th GW, but the level of lncRNA exhibited a decreasing trend from the peak at the 20th GW. In contrast, mRNA levels began to decrease after reaching a peak at the 21st GW, and a small peak was observed at the 24th GW. Both SYT9 IncRNA and mRNA were highly expressed after the 33rd GW. There was a positive correlation between SYT9 lncRNA and mRNA (Fig. 4e). The expression of SYT15 mRNA and lncRNA in the 19th25th GW had similar fluctuations, and these were similar in the later days of pregnancy (Fig. 4f). Except for SYT9, five other proteins were detected in the plasma EXs with gestational fluctuation.

\section{GEPs of SVAT-IncRNAs and SVAT-mRNAs in pregnancy complications}

To explore the effect of pregnancy complications on lncRNA-mRNA in maternal circulating EXs, 38 cases of sPTB (28-36 GW), 19 cases of PE (28-36 GW), and 34 cases of GDM (28-39 GW) were studied. The lncRNA and mRNA expression of SLC18A2, SYT15, STX8, SV2C, $S Y T 9$, and $S Y P$ genes were detected by quantitative PCR. The results showed that SLC18A2 lncRNA and mRNA were higher in SPTB patients than in healthy pregnant women (Fig. 5a1), while STX8 lncRNA and mRNA were decreased (Fig. 5c1). In contrast, the expression of $S Y P$ lncRNA was increased (Fig. 5f1). The remaining molecules were not expressed differently between the SPTB and healthy pregnancy groups (Fig. 5b1, d1, e1, f1). In the
PE group, except for decreased STX8 lncRNA and STX8 mRNA (Fig. 5c2), there were no significant differences in gene expression for the remaining genes (Fig. 5a2, b2, d2, e2, f2). Interestingly, $S V 2 C$ lncRNA and mRNA, as well as SYP mRNA, were increased in patients with GDM compared with the healthy pregnancy group (Fig. 5d3, f3). No differences in gene expression were detected between the patients with GDM and the healthy pregnancy group (Fig. 5a3, b3, c3, e3, f3).

\section{Discussion}

This is the first study to demonstrate a connection between fetal-derived EXs and SVAT-associated lncRNAs (i.e., SYT15, SYT9, SYP, SLC18A2, SV2C and STX8; Table 1).Based on the results of this study, alteration of the IncRNA genes exists in the peripheral blood EXs of children with ASD. The expression pattern was also implied into analysis of adverse gestational outcomes, such as SPTB, PE, and GDM, although a larger size of clinical samples is required to calculate the range of lncRNA expressions in healthy newborns in relation to their health status.

SVATs are found in the CNS and peripheral circulation, playing an important role for SV release and recycling. In our previous genome-wide study of peripheral blood from children with ASD, the GEPs of lncRNAs as well as of mRNAs were identified to be differentially expressed in 12 SVATs loci [16]. The abnormal expression of SVATs often indicates abnormal development 


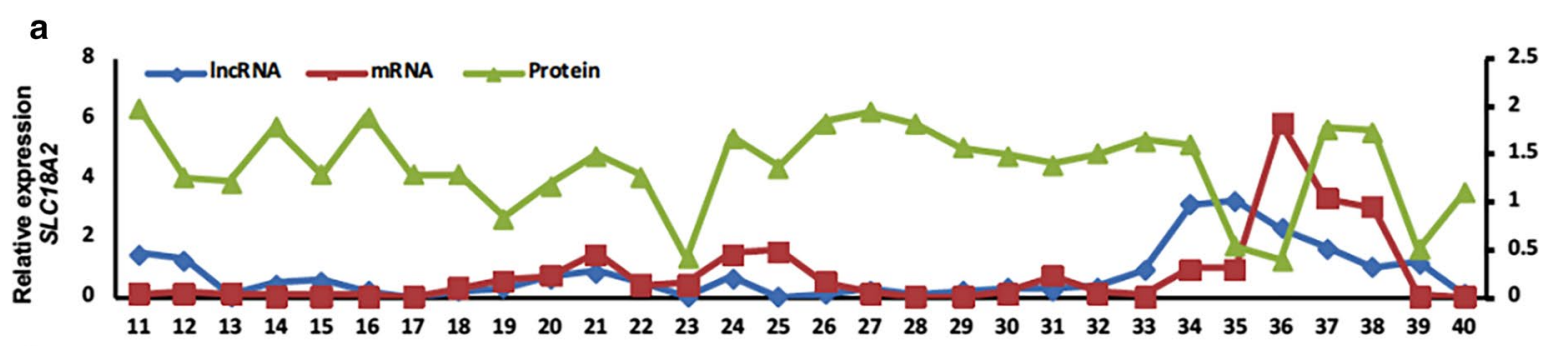

b

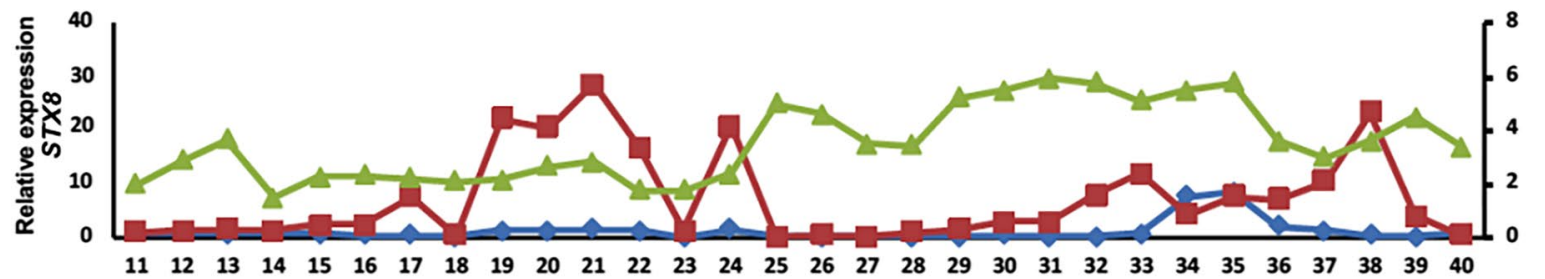

C

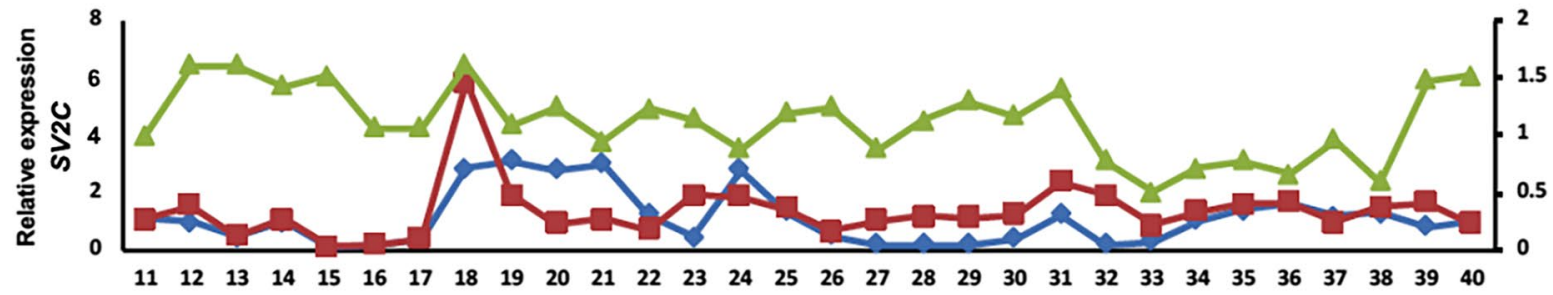

d

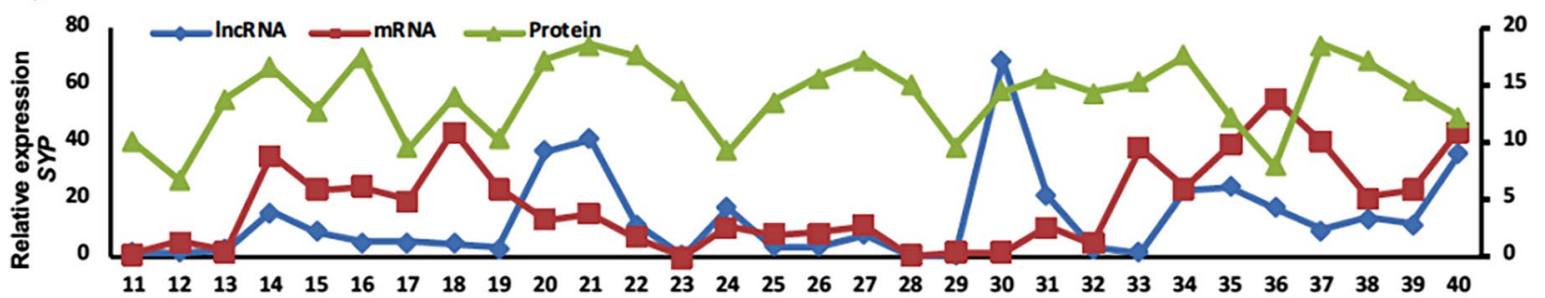

e

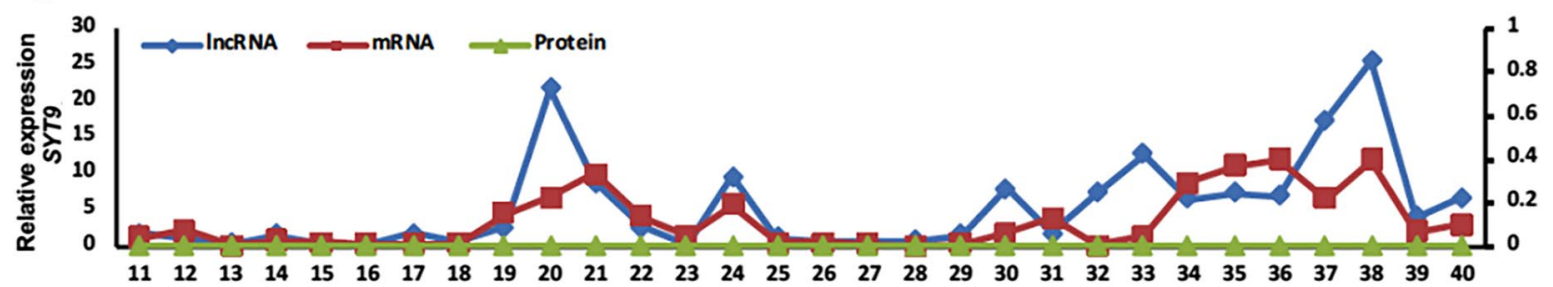

f

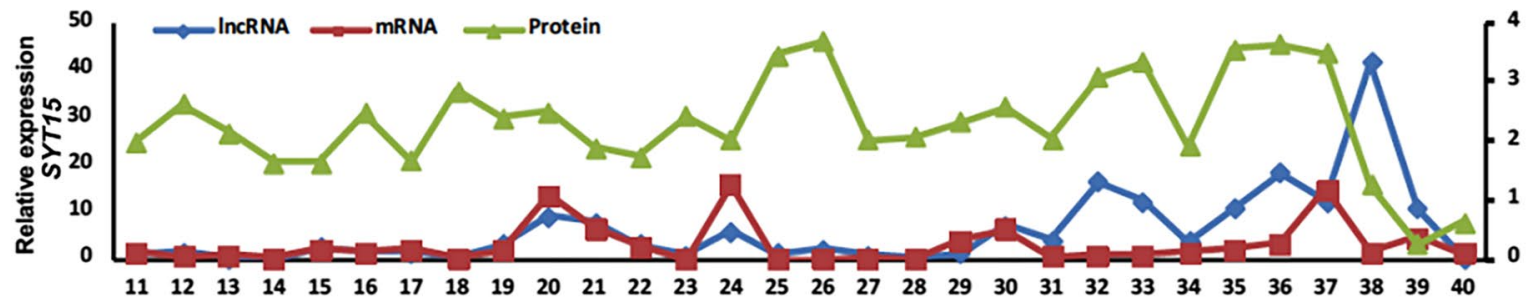

Fig. 4 Longitudinal assessment of GEPS. Six SAVT-associated IncRNAs (blue line), mRNAs (red line), and proteins (green line) were analyzed from the 11th-40th GW during physiologic pregnancies. The GEP patterns were based on exosomal pools collected independently at every gestational week with 30 unrelated pregnancies 


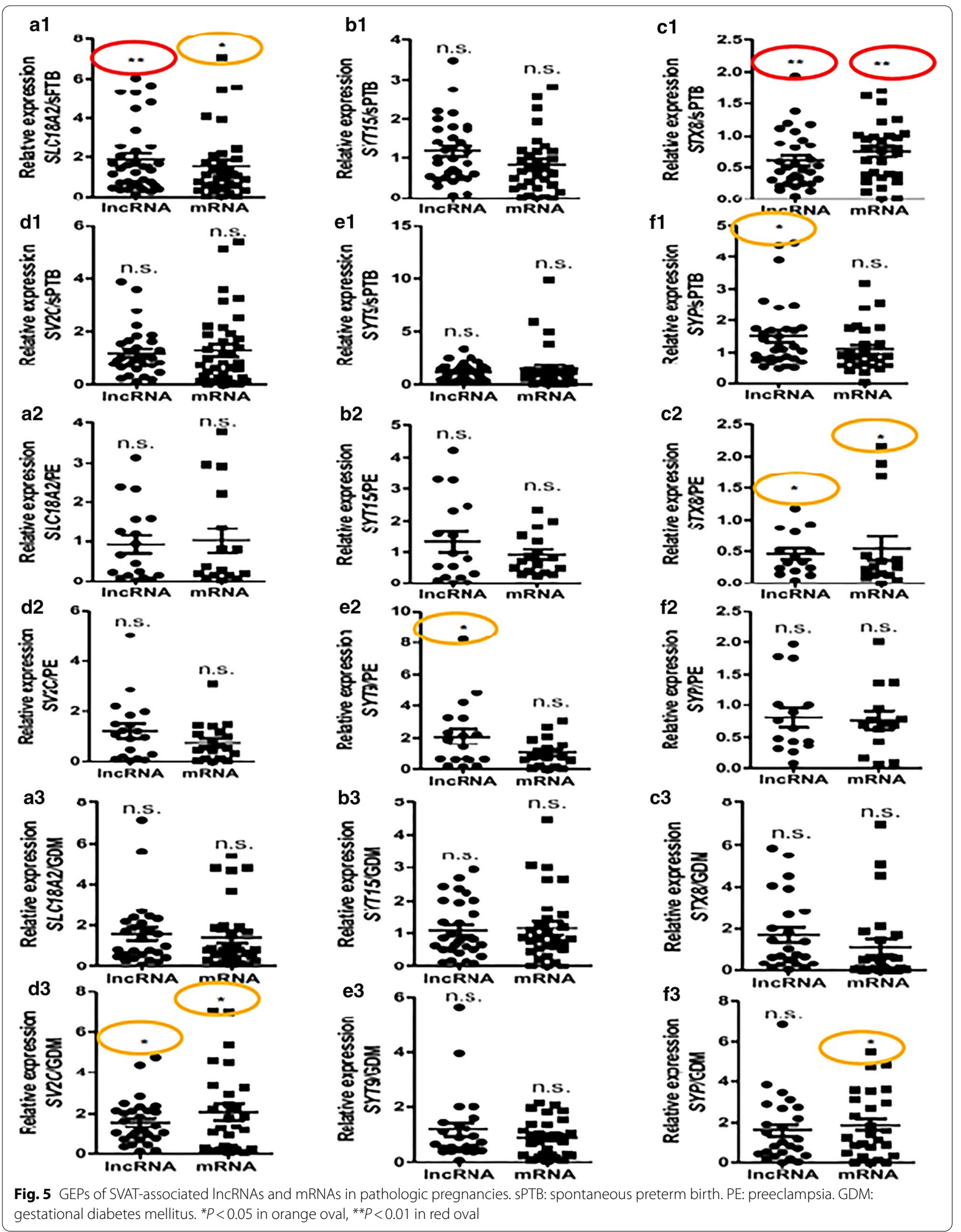


and an abnormal state of the nervous system. Abnormal expression profiles of SVATs are found in psychiatric disorders, such as autism, epilepsy, and schizophrenia [17]. Most of these disorders pose hidden risks during embryonic development. The perfection of fetal brain function during pregnancy involves a very precise process of SV cycling. EXs derived from neurons enter the circulatory system through the blood-brain barrier, including the EXs originating from fetal brain nerves. We know that EXs carry a variety of RNA, protein, and lipid molecules for long-distance signal transmission, whereas the characteristics of SVATs detected in EXs from peripheral blood remain unknown. It is a challenge to detect the functional state of neural circuits in the brain under living conditions. There is currently no effective way to respond to brain circuit function. EXs can carry $A \beta$ proteins that are transmitted between different neurons via synapses in patients with Alzheimer's disease [18]. The extensive activity of EXs in the nervous system suggests that EXs may be an important tool. In our study, EXs from maternal peripheral blood sources were shown to express NES and L1CAM, indicating neuronal-derived EXs. NES is primarily regarded as a marker of neural stem/progenitor cells. NES-positive cells are localized to tissue- or organ-specific sites in adults, where they serve as a quiescent resource of cells capable of proliferation, differentiation, and migration after re-activation [19]. The re-expression or upregulation of NES was observed in various tissues during repair processes after several types of injury, including the following: in reactive astrocytes after CNS damage [20]; during the fibrotic response to ischemic heart disease [21]; and in the context of mesangial cell repopulation after induced nephritis [22]. Therefore, most of the NES detected in healthy pregnant women is derived from embryonic neural precursor cells. L1CAM in neurons is essential in the neuronal development of mice, where L1CAM governs neuronal outgrowth, migration, and axonal guidance [23]. L1CAM is a transmembrane protein belonging to the immunoglobulin superfamily that interacts with several ligands, such as integrins, neuropilins, contactin 1 and 2, and CD24, as well as with the shed form [24]. In disease-free adults, L1CAM was observed exclusively in kidney epithelia [25]. Although we cannot rule out that the EXs are secreted by maternal neurons, EXs still contain fetal neurons. Through the assessment of the maternal neurologic function, the detection of abnormalities in pregnant women can also assess the synaptic function of fetal brain neurons.

Membrane-bound and secretory proteins are modified in the endosome reticulum (ER) and are essential for proper function of proteins by folding [26]. Previous studies have established that early endosomes arise from primary endocytic vesicles, which fuse with each other to form a larger endocytic structure [27]. It is likely that endocytic vesicles containing internalized EXs fuse with endocytic vesicles containing different cargoes and during endosome maturation towards the generation of intraluminal nanovesicles, which result in late endosome sheltering of endogenous vesicles and EXs taken up from another cell. It can be assumed that most of the internalized EXs are used by the cell by undergoing lysosomal degradation or recovery of some components via the trans-Golgi network [28].

The results in this study showed that PE and GDM did not affect the expression of SLC18A2 lncRNA and mRNA (Fig. 5a2, a3), indicating that these two pregnancy complications do not affect the function of the nervous system and the monoamine transport pathway. SLC18A monoamine 2 is a vesicular monoamine transporter that acts to accumulate cytosolic into SVs, using the proton gradient maintained across the synaptic vesicular membrane. Dysfunction in the activity of the monoaminergic system has been implicated in several human neuropsychiatric disorders [29] and is known to influence cognitive outcomes after traumatic a brain injury [30].

SYT15 and SYT9 encode the synaptotagmin family of membrane trafficking proteins, which are involved in the trafficking and exocytosis of secretory vesicles [31]. The STX8 gene is involved in protein trafficking from earlyto-late endosomes via vesicle fusion and exocytosis. The STX8 gene encodes a vesicle trafficking protein that functions in the early secretory pathway, possibly by mediating retrograde transport from cis-Golgi membranes to the ER [32]. The $S V 2 C$ gene encodes synaptic vesicle glycoprotein $2 \mathrm{C}$, which regulates the secretion of neural and endocrine cells. The $S V 2 C$ gene selectively enhances low-frequency neurotransmission and positively regulates vesicle fusion by maintaining the readily releasable pool of secretory vesicles [33]. The SYP gene encodes the synaptic protein, synaptophysin, an integral membrane protein of small SVs in the brain and endocrine cells. Synaptophysin is a calcium ion-binding protein that may also bind cholesterol and is thought to be involved in direct targeting of vesicle-associated membrane protein 2 (synaptobrevin) to intracellular compartments [34].

SPTB is a leading cause of newborn deaths and the second leading cause of deaths worldwide among children $<5$ years of age [35]. Many studies have investigated the etiology of SPTB, but fewer studies have investigated the consequence of brain damage in children born preterm. In this study a method for detecting maternal lncRNAs and mRNAs in EXs was developed to determine the influence on neuronal function. We found a significant effect of SLC18A2 and STX8 in SPTB compared with controls. SPTB may affect the function of SVs and hence the 
transmission of synaptic signals, thus causing nervous system dysfunction via SLC18A2 and STX8. Hypertension during pregnancy is the major cause of maternal and fetal morbidity and death. The most severe form is PE, which occurs in $6-8 \%$ of all pregnancies [36]. In many epidemiologic studies, PE is associated with an increased onset of cardiovascular and metabolic diseases in the later lives of the offspring [37]. The presence of PE during fetal life can lead to an increased risk of chronic noncommunicable diseases in later life [38]. The effect of PE on the development of synaptic transmission in the fetal nervous system is not known. Our results showed that pregnant women with PE have an abnormal expression of STX8 lncRNA and mRNA, while the exact correlation between further examination. The rate of GDM has been increasing, but little is known about the long-term complications of GDM in offspring. Evidence from epigenetic studies strongly advocates for the need to identify neuropsychiatric complications among offspring who are exposed to GDM. Several researchers have suggested that there is a possible association between in utero exposure to GDM and ASD among offspring [39]. In GDM patients, the expression of $S V 2 C$ lncRNA/mRNA and of SYP mRNA was higher than healthy pregnant women (Fig. 5d3, f3), indicating that GDM affects the release of fetal SVs through the SV2C and SYP pathways.

\section{Limitations}

A challenge of this study was that the total EXs we isolated from the maternal circulation were mainly derived from trophoblasts. Although neuronal markers (LICAM and NES) have been shown to be present within the total EXs, the portion of neuronally-derived EXs in peripheral circulation is unknown. Obtaining neuronal EXs as derived from fetuses would be even more difficult. This has limited application of SVATs as the specific biomarker for prenatal screening and risk assessment for autism or other neurologic disorders during pregnancy.

\section{Conclusions}

In summary, our results documented that exosomal lncRNAs are correlated with corresponding mRNA in healthy pregnant women. EXs can cross the bloodbrain barrier and hence can serve as a marker of brain health. Thus, IncRNAs that are associated with neurologic development found in EXs can be detected in plasma. Indeed, this work demonstrated exosomal presynaptic-related lncRNAs and mRNAs in blood plasma of pregnant women. The early manifestations of abnormal molecular contents often have potential early warning effects. Examination of the expression patterns of VASTs in maternal EXs during pregnancy and tracking their changes may provide reference for understanding the early development of neural circuits in fetal brain and provide valuable information for assessment of the brain function after birth.

\section{Abbreviations}

SV: Synaptic vesicle; SVATs: SV-associated transcripts; GEP: Gene expression profile; ASD: Autism spectrum disorder; GW: Gestational week; sPTB: Spontaneous preterm birth; PE: Preeclampsia; GDM: Gestational diabetes mellitus; EXs: Exosomes; SVAPS: Synaptic vesicle-associated proteins; SVC: Synaptic vesicle cycling; sPTL: Spontaneous preterm labor; pPROM: Preterm premature rupture of membrane; DEPS: Differential expression profiles; NTA: Nanoparticle tracking analysis.

\section{Supplementary Information}

The online version contains supplementary material available at https://doi. org/10.1186/s12967-021-02821-6.

Additional file 1: Table S1. Primers used for qRT-PCR.

\section{Acknowledgements}

We are grateful to our laboratory staff at the Sino-US Center of Translational Medicine for Development Disabilities for their technical support. We would like to thank faculty members and nurses in the Department of Obstetrics and Gynecology at the Nanfang Hospital for their assistance in recruiting patients.

\section{Authors' contributions}

NZ conceived and designed the project study. YF and NZ conceived and designed experiments. YF and CW performed experiments. YF, CW, ZW, JP, and MZ collected samples. YF and NZ wrote, and NZ finalized, the manuscript. All authors read and approved the final manuscript.

\section{Funding}

This work was supported in part by the Bureau of Shunde District Science and Technology under Grant (\# 2015CXDT06), Guangdong Provincial Department of Science and Technology under Grant (\# 2015B050501006), the National Institutes of Health (R01HD094381), and the New York State Office of People With Developmental Disabilities (OPWDD).

Availability of data and materials

All data generated or analyzed during this study are included in this published article and its supplementary information files.

\section{Declarations}

Ethics approval and consent to participate

This study was approved by the Medical Ethics Committee of Guangdong Triple-Nine Brain Hospital and of Nanfang Hospital of Southern Medical University. Informed consent was obtained from patients of pregnant women or guardians of children.

\section{Consent for publication}

Not applicable.

\section{Competing interests}

The authors declare that they have no competing interests.

\section{Author details}

${ }^{1}$ Department of Obstetrics and Gynecology, Nanfang Hospital, Southern Medical University, 1838 North Guangzhou Avenue, Guangzhou 510515, China. ${ }^{2}$ Sino-US Center of Translational Medicine for Development Disabilities, Southern Medical University, Guangzhou 510515, China. ${ }^{3}$ Department of Psychiatry, Guangdong 999 Brain Hospital, Guangzhou 510510, China.

${ }^{4}$ Department of Human Genetics, New York State Institute for Basic Research in Developmental Disabilities, 1050 Forest Hill Rd, Staten Island, NY 10314, USA. 
Received: 17 December 2020 Accepted: 9 April 2021

Published online: 15 April 2021

\section{References}

1. Hansel C. Deregulation of synaptic plasticity in autism. Neurosci Lett. 2019;688:58-61.

2. Otto KJ, Schmidt CE. Neuron-targeted electrical modulation. Synthetic Biol. 2020;367(6484):1303-4.

3. Martin EA, Lasseigne AM, Miller AC. Understanding the molecular and cell biological mechanisms of electrical synapse formation. Front Neuroanat. 2020;14:12.

4. Pereda AE. Electrical synapses and their functional interactions with chemical synapses. Nat Rev Neurosci. 2014;15(4):250-63.

5. Bonnycastle K, Davenport EC, Cousin MA. Presynaptic dysfunction in neu rodevelopmental disorders: Insights from the synaptic vesicle life cycle. J Neurochem. 2020.

6. Gamache TR, Araki Y, Huganir RL. Twenty years of SynGAP research: from synapses to cognition. J Neurosci. 2020;40(8):1596-605.

7. Langlete P, Krabberod AK, Winther-Larsen HC. Vesicles from vibrio cholerae contain AT-Rich DNA and shorter mRNAs that do not correlate with their protein products. Front Microbiol. 2019;10:2708.

8. Boen JRA, Gevaert AB, De Keulenaer GW, Van Craenenbroeck EM, Segers VFM. The role of endothelial miRNAs in myocardial biology and disease. J Mol Cell Cardiol. 2020;138:75-87.

9. Dong L, Lin W, Qi P, Xu MD, Wu X, Ni S, et al. Circulating long RNAs in serum extracellular vesicles: their characterization and potential application as biomarkers for diagnosis of colorectal cancer. Cancer Epidemiol Biomarkers Prev. 2016;25(7):1158-66.

10. Rusconi F, Battaglioli E, Venturin M. Psychiatric disorders and IncRNAs: a synaptic match. Intern J Mol Sci. 2020;21(9):3030

11. Liu M, Zhu H, Li Y, Zhuang J, Cao T, Wang Y. Expression of serum IncRNAXist in patients with polycystic ovary syndrome and its relationship with pregnancy outcome. Taiwan J Obstet Gynecol. 2020;59(3):372-6.

12. Jonas K, Calin GA, Pichler M. RNA-binding proteins as important regulators of long non-coding RNAs in cancer. Int J Mol Sci. 2020;21(8):2969.

13. Varcianna A, Myszczynska MA, Castelli LM, O’Neill B, Kim Y, Talbot J, et al. Micro-RNAs secreted through astrocyte-derived extracellular vesicles cause neuronal network degeneration in C9orf72 ALS. EBioMedicine. 2019;40:626-35.

14. Mustapic M, Eitan E, Werner JK Jr, Berkowitz ST, Lazaropoulos MP, Tran J, et al. Plasma extracellular vesicles enriched for neuronal origin: a potential window into brain pathologic processes. Front Neurosci. 2017;11:278.

15. Goetzl L, Darbinian N, Goetzl EJ. Novel window on early human neurodevelopment via fetal exosomes in maternal blood. Ann Clin Transl Neurol. 2016;3(5):381-5

16. Wang Y, Zhao X, Ju W, Flory M, Zhong J, Jiang S, et al. Genome-wide differential expression of synaptic long noncoding RNAs in autism spectrum disorder. Transl Psychiatry. 2015;5:e660.

17. John A, Elise N, Nancy H, Diandra B, Kate B. The neurodevelopmental spectrum of synaptic vesicle cycling disorders. J Neurochem. 2020.

18. Polanco JC, Li C, Durisic N, Sullivan R, Gotz J. Exosomes taken up by neurons hijack the endosomal pathway to spread to interconnected neurons. Acta Neuropathol Commun. 2018;6(1):10.

19. Wiese C, Rolletschek A, Kania G, Blyszczuk P, Tarasov KV, Tarasova Y, et al. Nestin expression-a property of multi-lineage progenitor cells? Cell Mol Life Sci. 2004;61(19-20):2510-22.

20. Gilyarov AV. Nestin in central nervous system cells. Neurosci Behav Physiol. 2008;38(2):165-9.
21. Calderone A. Nestin + cells and healing the infarcted heart. Am J Physiol Heart Circ Physiol. 2012;302(1):H1-9.

22. Daniel C, Albrecht H, Ludke A, Hugo C. Nestin expression in repopulating mesangial cells promotes their proliferation. Lab Invest. 2008;88(4):387-97.

23. Rathjen FG, Schachner M. Immunocytological and biochemical characterization of a new neuronal cell surface component (L1 antigen) which is involved in cell adhesion. EMBO J. 1984;3(1):1-10

24. BrummendorfT, Kenwrick S, Rathjen FG. Neural cell recognition molecule $\mathrm{L} 1$ : from cell biology to human hereditary brain malformations. Curr Opin Neurobiol. 1998:8(1):87-97.

25. Pechriggl EJ, Concin N, Blumer MJ, Bitsche M, Zwierzina M, Dudas J, et al. L1CAM in the early enteric and urogenital system. J Histochem Cytochem. 2017;65(1):21-32

26. Kawada K, Mimori S. Implication of endoplasmic reticulum stress in autism spectrum disorder. Neurochem Res. 2018;43(1):147-52.

27. Huotari J, Helenius A. Endosome maturation. EMBO J. 2011;30(17):3481-500.

28. Hu Y-B, Dammer EB, Ren R-J, Wang G. The endosomal-lysosomal system: from acidification and cargo sorting to neurodegeneration. Transl Neurodegen. 2015;4(1):18

29. Peter D, Finn JP, Klisak I, Liu Y, Kojis T, Heinzmann C, et al. Chromosomal localization of the human vesicular amine transporter genes. Genomics. 1993:18(3):720-3.

30. Markos SM, Failla MD, Ritter AC, Dixon CE, Conley YP, Ricker JH, et al. Genetic variation in the vesicular monoamine transporter: preliminary associations with cognitive outcomes after severe traumatic brain injury. $J$ Head Trauma Rehabil. 2017;32(2):E24-34.

31. Chen C, Jonas P. Synaptotagmins: that's why so many. Neuron. 2017;94(4):694-6.

32. Thoreau V, Bergès T, Callebaut I, Guillier-Gencik Z, Gressin L, Bernheim A, et al. Molecular cloning, expression analysis, and chromosomal localization of human syntaxin 8 (STX8). Biochem Biophys Res Commun. 1999;257(2):577-83.

33. Benoit RM, Frey D, Hilbert M, Kevenaar JT, Wieser MM, Stirnimann CU, et al. Structural basis for recognition of synaptic vesicle protein $2 \mathrm{C}$ by botulinum neurotoxin A. Nature. 2014;505(7481):108-11.

34. Ozcelik T, Lafreniere RG, Archer BT 3rd, Johnston PA, Willard HF, Francke U, et al. Synaptophysin: structure of the human gene and assignment to the X chromosome in man and mouse. Am J Hum Genet. 1990;47(3):551-61.

35. Langmia IM, Apalasamy YD, Omar SZ, Mohamed Z. Progesterone Receptor (PGR) gene polymorphism is associated with susceptibility to preterm birth. BMC Med Genet. 2015;16:63.

36. Jacquemyn Y, Zemtsova O. Risk factors and prediction of preeclampsia. Acta Clin Belg. 2010;65(1):1-12.

37. Perak Amanda M, Lancki N, Kuang A, Labarthe DR, Allen NB, Shah SH, et al. Associations of maternal cardiovascular health in pregnancy with offspring cardiovascular health in early adolescence. JAMA. 2021;325:658-68

38. Stojanovska V, Scherjon SA, Plosch T. Preeclampsia as modulator of offspring health. Biol Reprod. 2016;94(3):53.

39. Nahum Sacks K, Friger M, Shoham-Vardi I, Abokaf H, Spiegel E, Sergienko $\mathrm{R}$, et al. Prenatal exposure to gestational diabetes mellitus as an independent risk factor for long-term neuropsychiatric morbidity of the offspring. Am J Obstet Gynecol. 2016;215(3):3801e1-e7.

\section{Publisher's Note}

Springer Nature remains neutral with regard to jurisdictional claims in published maps and institutional affiliations. 